\title{
Thermal stability of premature infants during routine care under radiant warmers
}

\author{
John H Seguin, Robert Vieth
}

\begin{abstract}
The body temperatures of infants weighing less than $1500 \mathrm{~g}$ under radiant warmers during routine care were documented in the first week of life. Ten infants (median gestational age 28 weeks, median birthweight $913 \mathrm{~g}$ ) were studied. During 30 nursing interventions (mean $9 \cdot 2$ minutes) mean oesophageal and foot temperature changed $0^{\circ} \mathrm{C}$ and $-0 \cdot 11^{\circ} \mathrm{C}$, respectively.
\end{abstract}

A radiant warmer may limit heat loss during interventions because of easy access and rapid radiant warmer responsiveness.

(Arch Dis Child 1996; 74: F137-F138)

Keywords: radiant warmers, prematurity, body temperature.

Thermal homeostasis is important yet problematic for the growth and survival of very low birthweight (VLBW) infants $(<1500 \mathrm{~g})$. The efficacy of incubators and radiant warmers is based on rigidly controlled, static, and time limited research conditions. Their effectiveness under clinical conditions has not been adequately assessed. This report documents the body and environmental temperatures of VLBW infants in the first week of life who were under radiant warmers as part of routine care.

\section{Methods}

VLBW infants cared for under radiant warmers in the intensive care units at the University of Kansas Medical Center and Columbus Children's Hospital were eligible for entry into this study. Exclusion criteria included major cardiovascular or central nervous system problems or lack of parental consent. This study was approved by the Human Subjects Committee of each institution.

Ten infants were studied at a median postnatal age of 1.9 days (range $0.3-8$ days). Two infants were studied on two consecutive days. Median gestational age was 28 weeks (range 24-31 weeks), median birthweight $913 \mathrm{~g}$ (range 724-1356 g). All infants were cared for on Airshields Model PM-78 radiant warmers (Airshields, Hatboro, Philadelphia). Eight of the 10 were ventilated and covered with a plastic heat shield, either a Gaymar Neo-shield (Gaymar Industries, Inc, Orchard Park, New York) or Reynolds 914 film (polyvinyl chloride, 55 gauge; Reynolds Metals Co, Richmond, Virginia). These materials did not touch the infants. Supplemental humidity was added under the plastic covering of five with
$2 \mathrm{~cm}$ in diameter plastic tubing connected to gas heating and humidification units. Two infants rested on circulating warm water pads. Body temperatures were measured with thermistors placed on the mid abdomen, mid back, sole of one foot, and in the oesophagus. The surface thermistors were secured with a gel adhesive probe cover with foil backing to reduce radiant heat gain. Air temperature near the infant was measured with a radiantly shielded thermistor. All thermistors were calibrated against a certified mercury thermometer in a water bath or air. Radiant warmer power consumption was measured with a watt-hour metre (model EM-5, Ohio Semitronics, Columbus, Ohio). The response time constant was instantaneous for five infants and one minute for five infants. Temperatures and watts were sampled each minute for six hours between 0900 and 1900 hours and stored in a computer spreadsheet format (details available on request).

A digital hygrometer (Fisher Scientific, Pittsburgh, Pennsylvania) measured relative humidity in the neonatal intensive care unit and close to the last five subjects. Two measurements were made during the protocol and recorded at the bedside. All nursing interventions were timed and recorded on a bedside log. Seventy five nursing interventions were logged during the 72 patient-hour recordings.

Differences between the oesophageal and foot temperatures at the conclusion of and the minute before each nursing intervention were calculated. These differences were then averaged and analysed by linear regression compared with birthweight and gestational age. Individual temperature changes were analysed by linear regression versus intervention duration. Temperature and radiant warmer wattage data were plotted against time and examined visually for trends.

\section{Results}

Thirty seven interventions of at least four minutes in length were grouped as 30 'short' interventions (mean 9.2 minutes, range 4-17) and seven 'long' interventions (median 30 minutes, range 20-118). Short interventions included vital signs, suctioning of the endotracheal tube, blood sampling, turning, and changing nappies. Long interventions included percutaneous central vein catheter placement, head ultrasonography, $x$ ray pictures, lumbar puncture, and multiple routine nursing procedures.

Mean oesophageal temperature difference was $0^{\circ} \mathrm{C}$ (range $-0 \cdot 1-+0 \cdot 09$ ) and mean foot temperature difference was $-0.11^{\circ} \mathrm{C}$ (range $-0.53-+0.64)$ for short interventions. Sixteen 


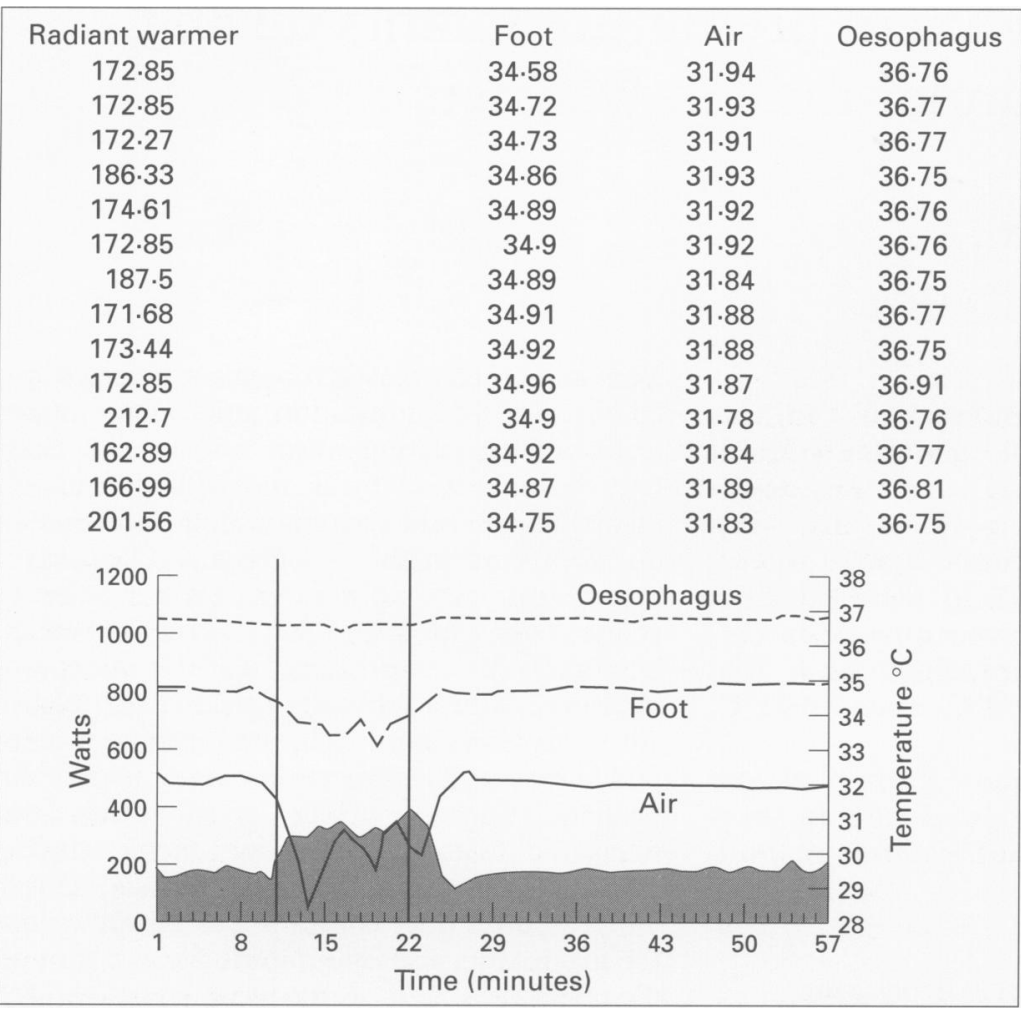

Figure 1 Example of body and air temperatures and radiant warmer power use (shaded area) compared with time during a 13 minute intervention on a 12 hour old infant ( 29 weeks gestational age; birthweight $843 \mathrm{~g}$ ) in supplemental humidity. of the 30 interventions were associated with foot temperature rise of $\geqslant 0 \cdot 2^{\circ} \mathrm{C}$. There was no correlation between the magnitude of body temperature change with birthweight or gestational age. There was no correlation between duration of intervention and oesophageal or foot temperature change. Figure 1 shows body and air temperature changes and radiant warmer wattage during a short intervention.

Mean oesophageal temperature difference was $-0.01^{\circ} \mathrm{C}$ (range $-0.33-+0.69$ ) and mean foot temperature difference was $-0.8^{\circ} \mathrm{C}$ (range $-2 \cdot 6-+2 \cdot 0$ ) for long interventions. Two long interventions resulting in increased foot temperature were investigated. One was preceded by subnormal body temperatures and the other was followed by continued rise of body temperatures to supranormal levels, both suggesting inappropriate servo control.

Relative humidity in the intensive care unit and next to five infants was measured during the recording period. The mean (range) was $30 \%(19 \cdot 5-42)$ in the unit, $51 \cdot 7 \%(25-82)$ around three infants receiving added humidity, and $27 \%(24-31)$ around the two unsupplemented infants. The mean difference in relative humidity between the intensive care unit and the infant environment for those with and without added humidity was $+21.7 \%$ and $-3 \cdot 8 \%$, respectively.

\section{Discussion}

We unexpectedly found small body temperature changes under radiant warmers during short interventions and moderate changes during long interventions. This was probably caused by the rapid and efficient increased radiant heat production in response to decreased skin temperature during interventions. A rapid return to pre-intervention temperatures was seen (figure 1). Both the radiant heat and the replacement of the heat shields and humidity could have contributed to this finding. The duration of the nursing interventions was also shorter than expected and may have aided temperature preservation.

Foot and oesophageal temperature increases were recorded with some interventions. Undetected partial dislodgement of the servo skin thermistor results in overheating in incubators and radiant warmers. Manual radiant heat control could cause the same effect, and is sometimes briefly used by nurses trying to compensate for anticipated increased heat loss.

Ducker $e t a l^{l}$ found more central-peripheral body temperature variance with skin servo than air servo control of incubators during the first two days of life. The effect of specific interventions on temperature was not addressed. Marks and Gotkiewicz presented preliminary data for body and environmental temperature changes during specific interventions in skin servo controlled incubators. ${ }^{2}$ They commented that the integrity of the servo probe was easily compromised. Mok et $a l^{3}$ showed that infants in air servo controlled incubators sustained significant and prolonged drops in body temperature during routine nursing interventions.

There are several differences between the conditions and results of Mok's study and those reported here. In the former, heat output increased only when the air temperature dropped. The environmental temperature of those infants decreased when the air temperature dropped and the bubble plastic blanket was removed for procedures. This and the slower response of a convective heating system could have caused the large temperature differences seen. Freer access to the infants may shorten procedure time and therefore reduce temperature declines, but comparisons of identical nursing tasks would be needed to confirm this. Finally, abdominal skin temperature may not be an accurate substitute for true core temperature. To illustrate this, we found that the mean (SD) differences between the abdominal and oesophageal temperature in two of our study infants over the course of the recording period were $-0.08^{\circ} \mathrm{C}(0.17)$ and $-0.22^{\circ} \mathrm{C}(0.25)$. The variability was too great to be of clinical value. Good correlation of rectal and prone abdominal skin temperature has been reported. 4

We conclude that if the hazards of skin servo control are avoided, care under a radiant warmer with use of ancillary thermal devices can attenuate thermal stress associated with interventions on VLBW infants.

We acknowledge support from the Children's Miracle Network Grant 89-10 (University of Kansas) and the Bremmer Foundation Grant 921011 (Ohio State University).

1 Ducker DA, Lyon AJ, Ross Russell R, Bass CA, McIntosh N Incubator temperature control: effects on the very low birthweight infant. Arch Dis Child 1985; 60: 902-7.

2 Marks $\mathrm{KH}$, Gotkiewicz EM. Thermoregulatory adjustments in preterm infants using a servo controlled double walled convection incubator. Ped Res 1991; 29: 225A.

3 Mok Q Bass CA, Ducker DA, McIntosh N. Temperature instability during nursing procedures in preterm neonates. instability during nursing proced

4 Dollberg S, Atherton HD, Allen K, Hoath SB. A transcutaneous alternative to rectal thermometry for continuous measurement of core temperature in preterm infants. Ped Res 1994; 35: 222A. 